\title{
Value-based lean implementation in a surgical unit: the impact of the methodology
}

\author{
Marc Sales and Rodolfo De Castro \\ Department of Business Organization, \\ Management and Product Design, Universitat de Girona, Girona, Spain
}

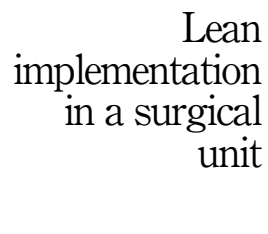

Received 3 November 2020 Revised 22 December 2020 Accepted 28 December 2020

\begin{abstract}
Purpose - This paper aims to present a method for strategic planning and implementation processes in health care based on lean management.

Design/methodology/approach - Within the framework of the action research methodology, the authors present the ten steps of a kaizen project, which enable lean transformation over a period of time. The study is underpinned by a literature review of enablers and barriers and an implementation case in a tertiary care hospital.

Findings - Key points and possible contingency issues are presented for each of the steps, and a successful lean tools intervention is illustrated by examples of improvement projects of the surgical process. Conclusions of the implementation establish a roadmap for improvement projects in hospital environments based on lean management, thus bridging the existing gap between the large number of theoretical projects (much of the projects described are not sustainable over time as the hospital sector is very particular) that have failed to be implemented, or been paused mid-term, and the self-sustaining projects developed by improvement teams in the hospital.

Originality/value - The study details knowledge gleaned from a three-year project entailing various stages: forming improvement teams; training health-care professionals in lean management; drawing up a process map to identify value stream mapping improvement opportunities; implanting projects and verifying the results obtained; and finally, laying the cornerstones, which would make the project self-sustaining and open to long-term continuous improvement.
\end{abstract}

Keywords Health care, Hoshin Kanri, Continuous improvement, Patient care, Surgical process, Value stream mapping, Kaizen

Paper type Research paper

\section{Introduction}

Production methods such as lean management have been used in hospitals since the early 2000s (Akmal et al., 2020; Barnabè and Giorgino, 2017; Bonome et al., 2016; D’Andreamatteo et al., 2015; Perona et al., 2016; Visintin et al., 2017).

The first reference to the term "Lean" was made by John Krafcik and subsequently used by Womack et al. (1990) to describe the steps taken in Toyota's production system to continuously improve its the efficiency and effectiveness by eliminating waste. Womack and Jones (1996) pointed out that to meet customer needs, an organization must first identify what customers perceive as value. The philosophy behind this methodological approach can be summarized in five lean principles: (1) define value from the point of view of the client, (2) work to eliminate steps in the process that do not add value, (3) create a continuous flow in the remaining steps, (4) implement flexible systems in places where the flow is not possible and (5) work continuously in the pursuit of perfection.

Bortolotti et al. (2015) define lean as "a managerial approach for improving processes based on a complex system of interrelated socio-technical practices." Increasingly, lean manufacturing practices are being adopted by companies to improve their competitiveness.

This research was possible thanks to the financial support from the grant ECO2017-86054-C3-3-R (MINECO/AEI/FEDER/UE).

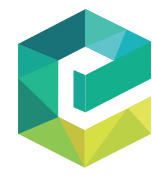

The TQM Journal (C) Emerald Publishing Limited 1754-2731
-2020-0249 
A lean management approach brings greater flexibility, lowers costs and improves the quality of the product, and even though originated in the automobile world, it is currently being used in an array of industries.

Both the manufacturing and service industries have many examples of organizations that have improved their competitiveness by implementing lean tools, particularly hospitals and health-care institutions (Hicks et al., 2015). Souza (de Souza, 2009) states that it is unclear when lean philosophy was first introduced in the health-care industry, but publications initially appeared in 2002. Nowadays, there is a very clear "need" to use lean's tools and techniques in health care to guarantee quality, patient safety, costs, waiting times and staff (Joosten et al., 2009; Bonome et al., 2016), and some of hospitals try to play by lean strategy tools for strategic planning and management, particularly in reference to the Hoshin Kanri policy deployment system and the "focus, alignment, integration, and review" method (Barnabè and Giorgino, 2017), or Kaizen-Kata approach, which uses using problem-solving or other methods (Suárez-Barraza and Miguel-Davila, 2020).

These types of projects are often unsuccessful in health care as a number of critical factors condition the success of lean implementation in health care: "management commitment and involvement," "training and education" and "employee participation and empowerment" (Netland, 2016).

However, the progress of lean is often found to be inhibited as medical professionals have failed to engage or provide clinical leadership in supporting the lean trajectory (Kahm and Ingelsson, 2020). This has resulted in limited outcomes, sustainability implications and failed projects (Lindsay et al., 2020). These factors, among others, were taken into account when creating the new method presented in this paper, which was successfully implemented in Vall d'Hebron Hospital. This contribution proposes an innovative way to implement improvements in scheduling processes in hospital operating theaters.

Managing an operation theater (OT) complex requires a considerable number of resources. These human, technological, material and care resources enable area managers to adapt the waiting list of their patients needing surgery to the hospital's management capacity.

Key factors affecting the coordination and efficiency of the surgery unit include the size of the OT complex, the number of services requiring coordination, the level of technology within the operating theaters, the discharge capacity of the hospital for patients undergoing surgery, the complexity of the surgery and patient flow. Our experience with various OT complexes has been useful to detect their basic needs and deliver efficient comprehensive surgical management solutions.

This paper sets out the specific needs detected in the surgical units of a tertiary care hospital over a three-year period and describes the efficient way in which they have been managed.

The objectives of the paper are as follows:

(1) To explain the context in which a hospital analyzes the process of assigning operations to operating theaters.

(2) To discuss and set out a management strategy designed and executed in a hospital case implementation.

The paper is structured as follows: firstly, in introduction, a literature review is contrasted with the actual case where the project was implemented. This is followed by materials and methods where it is presented the methodological study framed within the scope of action research at Vall d'Hebron Hospital in Barcelona, Spain. Next, in results, the authors' management implementation process is proposed. This is based on the ten steps of the standard work process of lean projects in a surgical process improvement team, using lean tools, particularly value stream mapping (VSM). Finally, we present the discussion of the implementation process step by step, followed by the conclusions. 
The literature on improvement projects in surgical areas highlights the underlying reasons for the difficulties involved in implementing lean projects (D'Andreamatteo et al., 2015); however, there is a notable lack of real cases where lean management has been implemented.

Akmal has detailed a set of case study articles describing the implementations of lean thinking in the health-care sector. A large number of these cases $(83 \%)$ used lean thinking as an improvement methodology, while other cases used lean six sigma. Departments and functions, such as emergency departments, medical records, radiology, pharmacy, endoscopy, inventory management and scheduling of operating theatres, were chosen more for lean initiatives, and the implementations went more smoothly within them (Akmal et al., 2020).

Most research shows a theoretical framework in which lean criteria and management tools are adapted for use in the area of health care (Bonome et al., 2016), but without translating the concepts into a language easily understood by health-care professionals. This leads to initial difficulties from the outset, as the people leading the improvement project fail to fully understand what needs to be done once the technician, who is usually an engineer, finishes their job and leaves the project in the hands of the hospital manager (Schattenkirk, 2012).

To be both successful and sustainable over time, lean implementation must meet three criteria: (1) commitment on behalf of the management, (2) training and education and (3) employee participation and empowerment (Netland, 2016). But, it is important to take into account the patient voice. As Gustavsson highlights, the view on patients within health care is being transformed from one based on servility to that of patients as customers. This approach appears to overcome a gap of relying solely on health-care professionals when identifying patients' need. For this reason, the patient's vision is taken into account when redesigning patient flows (Gustavsson et al., 2016).

However, for lean methods to be implemented successfully, two additional elements must be present: a culture of organization and a willingness to adopt "soft practices" (Bortolotti et al., 2015). If the implementation is limited to simply introducing lean tools, as it often is, the transformation will not be sustainable over time (Hu et al., 2015; Antosz and Stadnicka, 2017). There is no adherence to the new processes and protocols if they have not been implemented from the bottom up.

The improvement method presented in this case was inspired by the large number of recent publications on lean health care found in literature reviews (Bonome et al., 2016; D’Andreamatteo et al., 2015; Parkhi, 2019; Poksinska, 2010; Lima et al., 2020; Nicolay et al., 2012). Findings from these studies are outlined below.

According to D'Andreamatteo (D'Andreamatteo et al., 2015), 134 papers were selected from a total of 243 theoretical articles. These were analyzed to find the difficulties encountered, as well as keys to success when implementing lean projects in the health-care industry. However, very few articles are a comparative analysis of projects that have actually been implemented. Three main phases of a lean project can generally be identified in all of the articles: (1) an introduction to lean methods and the process of change, (2) implementing the project using lean methodology and other process improvement techniques and (3) a critical assessment of the project and the implementation process. The following factors are identified in the first and second phase: barriers and challenges to be achieved, success factors of the project, the implementation processes, sustainability and a framework for monitoring improvement.

Barriers to introducing lean methodology into the health sector affect the success of improvement projects. The literature review highlights several factors attributing to these barriers: the receptiveness of professionals, the complexity of introducing changes into the process, the fact of sharing information with professionals outside the process, the culture of change, the high variability of processes, the staff's understanding of the lack of lean

implementation in a surgical

unit 
methodology, problems defining what waste is and giving the project the correct focus. These factors play a critical role in slowing the shift toward lean processes within the health sector (Poksinska, 2010; de Souza and Pidd, 2011; Leite et al., 2020).

The success factors set out in the article mainly stem from the support given by level managers to introducing lean processes. This includes the level of leadership from the healthcare professionals themselves, including all staff on the improvement teams, and how well defined the circuits and procedures are (Poksinska, 2010).

Another fundamental aspect of success is being able to perform an exhaustive initial assessment. This means that the transformation of processes can be clearly and precisely visualized from the initial stages through to completion (even though continuous improvement is never-ending); thus, the overall improvement can be accomplished together with an achievable future strategy and credible indicators (Kollberg et al., 2006; Niemeijer et al., 2011).

Regarding the process of implementing lean projects, D'Andreamatteo et al. (2015) point out that lean management training for all health-care professionals is key. Staff need to be involved in all the processes, including introducing the concept of pilot testing. This enables temporary improvements to be implemented for learning and monitoring purposes (to see if expectations are met) through cocreation (Ingelsson et al., 2020) before implementing the proposals for improvement permanently, taking into account the global, long-term strategic objectives and sustainability of the plan. Lean management implementation is an entire transformation project, not simply a few stages in the process. When it is not taken as an entire process, the degree of success is limited, and practices tend to revert as they are not linked to previous or later processes (Machado and Leitner, 2010). According to Machado, where and the degree to which lean techniques are used greatly influence the success of the project. In our case, we believe that an operation complex is an ideal environment, because many process improvement needs are combined in a controlled environment.

How sustainable the project is will largely depend on current and future managers' leadership, and health-care professionals' degree of involvement, understanding and conviction regarding the new improvement processes (Papadopoulos et al., 2011; Burgess and Radnor, 2013).

A hospital is primarily an entropic system, which will always tend toward the most comfortable zone depending on the lean management processes employed. Despite being more efficient for the hospital and safer for the patient, these processes are not always the easiest to implement and maintain.

If this objective is not pursued, the processes may return to what they were before the lean transformation. The staff tend to forget the new employment policies because it is easier to go back to old habits, and so the change needs to be sustainable (Ben-Tovim et al., 2007). Lean transformation is never-ending, in that there are always things that need to be improved and waste that can be eliminated. The management should be aware of the need to support research and provide it, as well as implementing improvements and pursing perfection and long-term sustainability (Machado and Leitner, 2010).

Active monitoring of indicators will always be necessary to evaluate the current situation, and proposals for improving the new processes and creating real value for the patient and the professionals must continue to be made (Burgess and Radnor, 2013; Toussaint and Berry, 2013; Gustavsson et al., 2016).

Finally, the literature analysis reveals that most studies mention few real examples or provide information about the implementation process of lean projects that could help future studies to streamline the use of the lean methodology to the health sector (Bonome et al., 2016). In the article by Chung et al. (2017), a systematic review of projects is carried out in the intrasurgical field, where process mapping was used to identify steps that could be modified to improve the results, and a series of interventions was implemented for this purpose. 
Worthy of note is the article by Henrique et al. (2016) that focuses on the VSM methodology as a key tool for understanding the overall vision of the process in six phases, including its bottlenecks and waste sources. The objective of our work, however, is to expand the application framework and present a new case capable of creating VSM in the surgical process as a whole. This new implementation process takes the three main flows into consideration: the flow of patients, the flow of information and the flow of materials (Zidel, 2006).

\section{Materials and Methods}

Managing change in a hospital environment requires the participation of all health-care professionals and administration staff involved in the processes to be improved. Ben-Tovim et al. (2007) cited health-care professionals and administration staff in particular, but even porters and cleaning staff are key in the surgical processes.

The action research methodology was chosen, as this has been used on other occasions to investigate experiences in the health-care environment (Perona et al., 2016; Visintin et al., 2017). This led to employing a mixed-methods approach, which included participation in work teams and secondary data analysis and observation. Following on from Coughlan and Coghlan (2002), Voss et al. (2002) and Yin (2003), data were collected from mainly direct and participant observation, as well as documents, archives and interviews.

The author led the project team, which developed a ten-phase stages to standardize processes within an improvement team. The project was carried out over a three-year period in the surgical units of the hospital. The improvement teams included a total of 368 healthcare professionals who were organized into work teams of between 6 and 14 people. A 4-h basic training course in lean processes and methodology was given, and all the teams participated in four 2-h work sessions. Over the three-year implementation period, the teams carried out 80 improvement projects in the hospital's process department, specifically within the framework of surgical processes.

Action research methods establish four critical steps: diagnosing, planning action, taking action and evaluating action (Coughlan and Coghlan, 2002).

Following the steps in the methodology, the process team's common improvement goals were: elaborating and analyzing VSM within the process (diagnosis); detecting opportunities for improvement, field observation and a thorough study of the root causes of each problem (planning action); implementing improvement actions and piloting them to reach the expected indicators (taking action); and finally, evaluating the improvement outcomes (evaluation action).

The research has focused on standardizing surgical processes and implementing them in other hospital units and blocks. The work groups evolved into an improvement teams that routinely detected and resolved security-related incidents and prioritized new projects based on the hospital's strategy. The same work group may have implemented more than one improvement project in their surgical unit.

The kaizen activities focused on three large areas: improving of patient flow, improving internal communication between professionals and improving the material flow needed to support the process.

Vall d'Hebron University Hospital Campus in Barcelona is one of the largest hospital complexes in Spain, with over 9,000 professionals, 1,100 beds and a throughput of more than $1,200,000$ patients each year in all departments: outpatient care, diagnostic testing, emergency care, critical care units, surgery and hospitalization.

Approximately 38,000 surgical interventions are performed every year. More than 900 professionals work in the hospital's surgical block, which is divided into four independent units with a total of 50 operating rooms. Lean process improvement teams held over 450 
meetings, and more than 130 pilot tests and Kaizen actions were implemented in the various surgical units.

\section{Context of the action research}

This research was based on work carried out from the Department of Advanced Processes and within the framework of a project designed to improve surgical processes within the oncampus surgical units.

Currently, the Department of Advanced Processes is following the lean continuous improvement method to implement its own management model. This model is currently being extended to all areas and departments of the hospital.

At the end of 2018, the process department included eight engineers and five process owners (health-care professionals) who developed a program of improvement projects in all the surgical wards, emergency services and critical care units, as well as complementary services such as pharmacy, laboratory, maintenance, computer science, etc. In addition, a Clinical Practice Improvement Unit, an Innovation Unit and a Patient Safety Unit were responsible for notifying incidents detected along the patient flow.

In 2015, there were a great many variations regarding how the hospital's operating theaters were being allocated and used. The system was inefficient due to a lack of criteria and followed inherited historical patterns. Many operating theaters were under-utilizing their resources, while others were at saturation point. In the management model being followed, the hospital's resources were poorly matched to the demands of the waiting list for surgery, and it was almost impossible to comply with the guaranteed times set out by the Catalan Health Department.

Lean methodology was then adopted to create various working groups that analyzed the existing process map (VSM) and projected a future map detailing how work should be carried out in a new block to deal more efficiently with the growing demands of the patient waiting list. The groups were made up of professionals from all fields: doctors, nurses, porters, administrative staff and cleaners ... everyone who was representative for the process. This led to several teams being set up to improve the process and set objectives, which would enhance patient flow as well as safety and activity indicators.

\section{Results}

As discussed in the literature review, the two main reasons behind the failure of projects attempting to implement lean culture in hospitals are the following:

(1) The task of creating improvement projects is delegated to external consultancies that have no continuity in hospital strategy, nor are they able to supervise health-care professionals' adherence to new processes.

(2) There is no firm commitment to address the cultural change that lean transformation requires to be guaranteed in the long term.

In general, the lack of continuity in implementing lean methods in health projects is due precisely to the fact that an external technician needs to propose and model new processes for health care. This means that the vast majority of projects are viewed as being imposed by managers who usually have short management cycles (typical in the Spanish Public Health System), and most policies change when a new government is elected. This is one of the main difficulties, because such a profound transformation project as lean health-care process management can only be sustainable and enduring if it is guided by strong, consistent leadership. Substantial effort is required to convince health-care professionals to maintain 
changes to the processes in the long term (D'Andreamatteo et al., 2015; Lindsay et al., 2020). (Lindsay et al., 2020).

In a general health policy context, the lack of evidence for integration of lean projects indicates a possible lack of managerial understanding or a failure to understand the potential benefits of adopting a solution thinking about the system. Management of health-care organizations is complex by nature, and the immediacy of the pressure to achieve short-term health outcomes may inhibit the proper development of the cultural changes that are required. The future challenge of health-care leadership must, therefore, be to understand and promote the benefits of using lean (Akmal et al., 2020).

Management at hospital opened an Advanced Processes Department with a fully integrated team of industrial and biomedical engineers, as well as medical and nursing staff. The method was deployed in all hospital processes simultaneously (emergency care, critical care units, surgical blocks, hospitalization and other support services) to create a similar rhythm in the general patient flow.

The hospital already followed a process-oriented culture, but it had not been deployed with such force or with such ambitious long-term goals. Another success factor highlighted (and employed at the hospital) was transferring the language of industry to the health-care environment, respecting the indicators of efficiency, safety and the satisfaction of both patients and health-care professionals.

Staff responded very favorably as they were aware of the discharge variability that affected hospital processes and the potential waste of resources during surgical activity. They were aware of the need to share information with the internal engineers who would support the new work philosophy and also the need to center attention on the patient adopting the slogan "Always, the patient first."

\section{Implementation processes}

The main contribution of this article can be summed up in the research aims: to explain the context in which a hospital analyzes the process of assigning operations to operating rooms and to discuss and establish a management strategy designed for, and implemented in, a real case. We can confirm that lean transformation is based on two premises: having a process engineer as a member of the hospital staff and all the staff having a committed and determined approach to implementing lean methods (Schattenkirk, 2012).

Drawing on the improvement projects using a VSM as action research, and taking into account the literature on barriers and success factors in lean projects implementation, a new approach was developed. This is the implementation process proposed in this paper (Figure 1).

Change management in a hospital environment requires the participation of all the health and administration personnel involved in the improvement processes. In the implementation presented here, we worked with various improvement teams over a three-year period. We provided all staff with basic training in lean processes and concepts, and then continued with a program deploying all the lean tools necessary to ensure the success of the project.

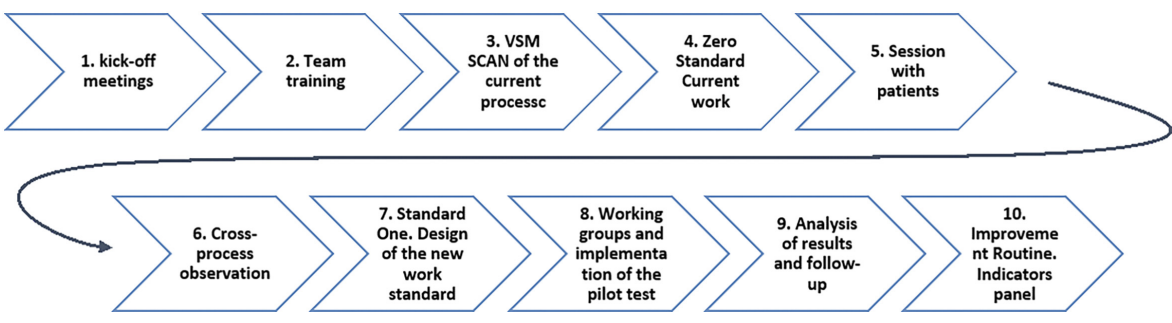

Figure 1.

The ten steps of the standard process of an improvement team

Lean implementation in a surgical unit 


\section{Discussion}

VSM is a key tool for understanding the overall vision of a process, detecting bottlenecks and waste sources. The main objective of our work was to expand the framework for application and present a new approach that can create VSM in the surgical process. The process takes into account the three main flows: that of patients, information and material (Zidel, 2006). The results were tested through a real implementation carried out in the surgical units. The aim was to contribute to the understanding of the key factors contributing to the success of the improvement project and its maintenance over time, as well as the barriers and restrictions that hinder it.

An improvement project usually takes an average of two months to implement and follows ten steps:

\section{Step 1 - kickoff meeting}

An initial meeting between the leaders or sponsors of the process (usually the head of a surgical service and a nursing supervisor), the process department engineers (who will support the project) and representatives from the health-care management team (who will ensure the success of the project).

In this first meeting, the scope of the project is agreed, expected results planned and the number of sessions required calculated. In addition, all the health-care professionals involved in the project are designated to improvement teams. The best days and times for staff to be free from their routine is also decided. This ensures they will be fully available to participate and bring value to the process group.

Step 2 - team training

Step 2 involves basic training in lean processes and methodology. Here, the group takes part in a "role play" workshop. A fictitious process is simulated and has to be optimized based on continuous improvement tools. During initial sessions, the need to adapt the language of engineering to the health-care environment is established, this makes the lean concepts more understandable and less aggressive from a health-care point of view. Process efficiency, increased safety and both patient and professional satisfaction are promoted.

This step in the training is essential to ensure the success of the project, as it is the best tool for all the staff to concur and understand the concepts of value contribution, process standardization, variability reduction and the role played by each person throughout in the process.

\section{Step 3 - value stream mapping scan}

The next step is constructing the VSM tool. The VSM or value flow process map is the main tool used to start each improvement group and each project. VSM was conceived by Rother and Shook (2003) in the industrial environment and is a visual management method that can analyze the process in its current state and design an ideal future state. This map includes both product flow and information and material flows. In our case, this is the series of events patients go through from the beginning to the end of the clinical process. Interaction between patients and health-care professionals (administrative staff, nurses, doctors or porters) can be visualized, and improvement opportunities in patient flow leading to an increase in perceived value from the patient's perspective can be detected. Thus, the risk of safety incidents will be reduced, improving efficient use of resources, avoiding wasted staff hours or materials and facilitating internal communication to manage the both the process itself and the health-care professionals' jobs.

This value flow map is the most important part of the project, as it allows each staff member to describe their contact with the patient and explain the purpose of their work. All 
professional interactions with the same patient are visualized, and current problems related to interruptions of flow, lack of communication or lack of preparation or availability of material resources are detected. Once these "problems" have been categorized and prioritized, they then become improvement opportunities and need to be reduced or eliminated when the future process map is drawn up.

As an example, the process within a surgical unit in outpatient surgery, the patient's journey (from beginning to end) is traced, from the reception of the waiting room to the surgical discharge, as it is shown in Plate 1.

Henrique (Henrique et al., 2016) pointed out that the entire multidisciplinary team can take part in drawing up the process map using a roll of paper hanging on the wall and colored postit notes to symbolize each phase of the process:

Post-its:

(1) Health-care professionals (green).

(2) Actions taken on patient (yellow).

(3) Problems/opportunities for improvement (pink).

(4) Information or material flow (blue).

All the health-care professionals involved in the process of constructing the VSM can see the tasks performed by their peers at a glance and also how their individual jobs interfere with patient flow. Using only this map, the group itself can detect a large number of improvement opportunities, as well as causes of potential safety incidents, which need to be handled.

The information obtained in the VSM is highly reliable and provides a basis for creating improvement projects, which can be used to draw up a new map of optimized processes.

Step 4 - standard zero

After constructing the VSM, it is essential that each professional is able to carry out their current standard work within a working day in most processes with a regular routine daily patient flow. Based on the effort-impact matrix, the sum of VSM and standard zero generates sufficient knowledge of the process to be able to identify priorities within the working groups. Standard work is defined as the most efficient method to produce a product or perform a service at a balanced flow, in this case, we call it standard zero, as this is the current situation of each professional's day-to-day. Using the future VSM, a new standard of work (version 1) can be designed. This is outlined in step 7 .

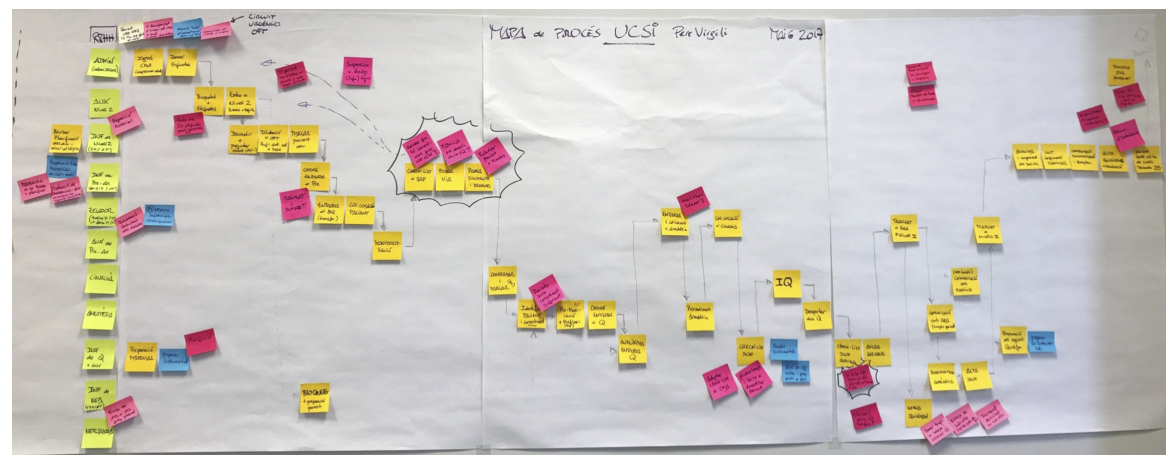

Plate 1.

VSM of the process at the outpatient surgery unit 
To create standard zero, each step of the process must be defined, and the work of each professional broken down into actions that follow a sequence and are organized and followed repeatedly in the same way. Any variation in the process can cause problems related to the safety or efficiency of the patient flow. Standard zero provides a baseline with which to develop a better approach. It promotes learning through continuous improvement methods, and if developed efficiently, should enable practically anyone to perform the work without any variation in the results.

\section{Step 5 - session with patients}

Before establishing priority work groups, and depending on the type of process, a session with expert patients is scheduled to glean insights from those directly affected by the process. An expert patient is a patient who has recently passed through the process and is willing to be interviewed and relate their experience, providing a critical and constructive viewpoint. In these sessions, patients often present a first-hand experience, which is completely different to that of the health-care professionals. This vision enables us to better understand how the patient experiences each step in the process (Gustavsson et al., 2016).

It is essential that the patients feel at ease when relating their experiences in these interview sessions, and that the medical staff who attended or operated on them are not present. These sessions are organized by the patient care department and the communication team, who draw up both an empathy and a journey map. The session is recorded and presented to the members of the improvement group and all medical staff involved in the process, so they are briefed about their patients' perceptions.

One such session involved patients undergoing major heart surgery in Vall d'Hebron. One of the main conclusions reached by the patients was that if they perceived a well-coordinated medical team, especially regarding information passed between staff, they felt that the team could be relied on. Clearly, a lack of coordination among staff generates distrust, fear and anxiety in the patient. These interviews confirm that work that can be coordinated through processes contributes to improving patients' perceptions of control, thus increasing their overall satisfaction with the service.

\section{Step 6 - cross-process observation}

It is not always possible to carry out these interviews with patients, but everyone must understand that they need to do a crossover observation of the patient flow drawn in the VSM. A cross-pointing observation occurs when the process improvement team moves to "Gemba" (meaning "the place where value is created"), and each member of staff steps into the shoes of another staff member, thus playing a different role to their usual one. For example, doctors observe the work of porters, porters observe nurses, nurses observe the work of administrators and so on.

These observations are usually set out in a "spaghetti" diagram (Plate 2). This shows the staff's movements throughout the day and lists interruptions to the observed person's work, as well as perceived opportunities for improvement. When staff are immersed in their routine activity, they are generally unaware of these opportunities.

When the observations have ended, the team meets to share their opinions of the existing process map and adds the new knowledge gleaned to the VSM of opportunities for

Plate 2.

Spaghetti diagram showing the movements of a surgical assistant over four hours in the new surgical unit

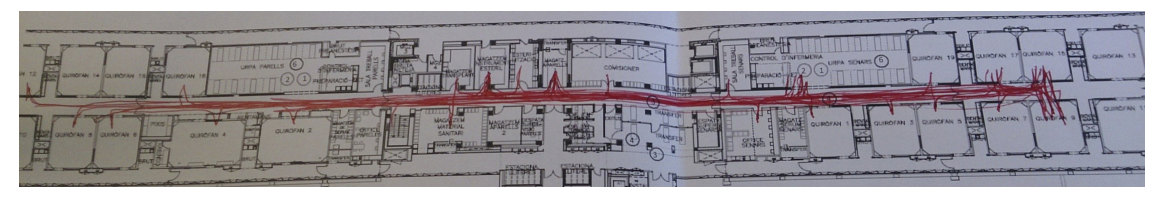


improvement. This task is essential to fully understand the problems associated with the process from the perspectives of all staff on the team.

Based on the author's experience in various process improvement projects, the most uncomfortable aspect for the team is invariably direct observation of another member of staff's work. Members of staff are neither used to being observed while carrying out their daily tasks nor are they used to observing others. Depending on the situation, this exercise can feel intrusive; therefore, this aspect needs to be foreseen, and everyone involved needs to be made aware of the importance of neutral and impartial observation. On observation days, professionals often do more tasks than usual, or behave slightly differently regarding their daily routine. It must be stressed that the person observing is not controlling or judging, but merely trying to understand how the process is normally carried out.

Step 7 - new standard

The next phase of the team process is to make a proposal for a new work standard, which reduces or eliminates the existing problems, and to create a new process map. Setting the new standard entails having an ideal VSM that attempts to solve some of the problems and to provide opportunities for improvement.

The new standard is closely linked to the task of setting up work teams for step 8, when pilot tests are carried out to check that the new standards lead to improvements and can be maintained over time. Each step in the process requires building new standards. These can be documented using a standardized sheet, or may be part of the protocol of action and/or communication between the health-care professionals, depending on the type of process.

Step 8 - work group and pilot test

The initial map generally proposes a large number of opportunities for improvement. Therefore, it is important to be able to divide them according to the impact they have on the process and in relation to the effort put in by the team. This is when priorities are identified regarding work to be carried out in the improvement groups and specific cases proposed for pilot testing where the suggested changes can be put into practice.

Four proposed scenarios form an effort-impact matrix are:

(1) Proposals with high impact and little effort (immediate priority for the process team).

(2) Proposals with high impact and great effort (priority conditioned by the resources available).

(3) Proposals with low impact and little effort (together with the group, it is agreed that this will be done internally, separate from the process team's schedule as these actions are usually based on staff's routines).

(4) Proposals with low impact and great effort (these are ruled out at this stage, but to be monitored in the follow-up routine, once the process team has become a continuous improvement team).

Bear in mind that in these types of improvement projects involving large teams of health-care professionals, rapid implementation of changes is sought to achieve "Quick Wins." Seeing results from the first stages of the project is the best way to keep the team motivated and persuade them to continue with their analyses.

The effort-impact matrix is a visual tool that helps prioritize the improvement groups to be formed from the process team.

Once the opportunities for VSM improvement have been detected, they are divided into three areas: process, communication and material, as each area requires a different approach to the projects. 
Following this, the projects are placed in the most appropriate scenario according to the impact of improving the process and the effort that the team or hospital itself (in terms of resources) needs to put into it.

Once all the projects are segmented, the group members are asked to assign themselves to one or two projects where they feel they could add more value or interest. Thus, various improvement teams are created, which include 2-3 members of the general task force. These teams meet over subsequent weeks to present proposals that can improve their particular process, and as a team, they decide on the best way to implement it.

For 2-3 weeks, the groups meet to analyze every opportunity for improvement, as well as the root cause of the problems and the best way to solve them so they do not reoccur, then the proposals are shared with the operations team. In this part of the process, it is important to follow the PDSA (Plan, Do, Study, Act) cycle, which is well used in the health care (Reed and Card, 2016).

The decision to do pilot testing (if required) is then taken. The results are then analyzed to see the impact of changes and whether it is feasible to make these changes permanent.

Pilot testing is one of the most complex parts of the process for the improvement team. This is when they have to confront the reality of the clinical process, explain procedures and train the rest of the staff in the operating theater to implement changes in the way they work or communicate with each other. This stage is, therefore, critical to the success of the project.

Step 9 - analysis of the results

In general, pilot testing usually shows very positive results in process improvement, as it enables the group to consolidate the changes made. However, if the results are not as expected, the team needs to rethink the origin of the problem and take a new approach to introducing the necessary changes. It should be understood that pilot testing is reversible, and routines can always go back to their initial state and be re-analyzed if deemed appropriate.

It is important to define the key performance indicators (KPIs) to be monitored and the degree of satisfaction expected from the health-care professionals involved.

This phase allows us to draw conclusions regarding the changes implemented and evaluate whether they can be maintained over time.

Step 10 - improvement routine

In summary, once the process teams have carried out the VSM, detected opportunities for improvement, carried out observation on the "Gemba" and studied the root causes of each problem in depth, then action is taken to improve and pilot them until the expected indicators are reached. From this moment onward, the process is standardized and implemented in all other surgical units. The operational work group involved in making changes to the process becomes in the end an improvement group focused on detecting safety incidents and finding solutions for them, as well as prioritizing new projects in line with the hospital management strategy, and improving indicators.

The ultimate goal is to achieve an improvement routine for each organizational process based on the criteria determined by the hospital's strategy, where the indicators in each area are monitored and in line with the priority set. The new improvement group members are responsible for ensuring safety, improving clinical practice and maximizing patient flow in the new process (Baldassarre et al., 2018).

Table 1 summarizes each of the ten stages and highlights the most significant observations such as actions, drivers and barriers. 


\begin{tabular}{|c|c|c|c|}
\hline \multicolumn{2}{|l|}{ Step } & \multirow{2}{*}{$\begin{array}{l}\text { Action/driver/barrier to highlight } \\
\text { Management's involvement is essential to motivating the project } \\
\text { team. Make the objectives clear before starting the procedure and agree on } \\
\text { the number of sessions that will take place }\end{array}$} & \multirow{3}{*}{$\begin{array}{r}\text { Lean } \\
\text { implementation } \\
\text { in a surgical } \\
\text { unit }\end{array}$} \\
\hline Step 1 & Kickoff meeting & & \\
\hline Step 2 & Team training & $\begin{array}{l}\text { Fundamental session for understanding the project. Adapt lean language to } \\
\text { the health sector. Explain the concepts of patient flow and interruptions or } \\
\text { bottlenecks }\end{array}$ & \\
\hline Step 3 & VSM scan & $\begin{array}{l}\text { All health-care professionals must be represented so that all points of view } \\
\text { can be visualized. Focus on the generic flow. Avoid going into details or } \\
\text { anecdotes. When a problem occurs, avoid blaming anyone. Instead, let } \\
\text { problems emerge so they become opportunities for improvement }\end{array}$ & \\
\hline Step 4 & Standard zero & $\begin{array}{l}\text { Monitoring the work of health-care professionals is not enough, their } \\
\text { routines need to be analyzed so that they can be adapted to the needs of } \\
\text { patient flow. If you question the work of other professionals, defining } \\
\text { standard zero can become a point of contention. Reaching an understanding } \\
\text { is paramount }\end{array}$ & \\
\hline Step 5 & Session with patients & $\begin{array}{l}\text { Patient selection is critical. Patients who view the process with a critical eye } \\
\text { add value to the group and should be sought. Avoid focusing on the } \\
\text { particular case of patient; focus on their perception of the process from their } \\
\text { viewpoint }\end{array}$ & \\
\hline Step 6 & $\begin{array}{l}\text { Cross-process } \\
\text { observation }\end{array}$ & $\begin{array}{l}\text { The fact that health-care professionals observe each other while carrying } \\
\text { out their work may seem violent and unpleasant, but it is very necessary to } \\
\text { fully perceive the responsibility of each professional throughout the } \\
\text { process. Those observed should not alter their routine or do more work than } \\
\text { they usually do, as this would distort reality }\end{array}$ & \\
\hline Step 7 & New standard & $\begin{array}{l}\text { This new way of working must lead to improvement for all the health-care } \\
\text { professionals involved. This does not mean overburdening some to free } \\
\text { others; it means balancing the tasks that need to be balanced and adding } \\
\text { value to both the health-care professional and the patient }\end{array}$ & \\
\hline Step 8 & $\begin{array}{l}\text { Work group and pilot } \\
\text { test }\end{array}$ & $\begin{array}{l}\text { Starting with a quick win is essential to motivate the whole group. Focus the } \\
\text { first project on actions that will make a big impact on results and patient } \\
\text { and professional satisfaction will relatively little effort. If the pilot task is } \\
\text { unsatisfactory, do not be discouraged. You can go back and rethink the } \\
\text { project. Use the concept of trial and error, and when the optimal result is } \\
\text { reached, standardize }\end{array}$ & \\
\hline Step 9 & $\begin{array}{l}\text { Analysis of the } \\
\text { results }\end{array}$ & $\begin{array}{l}\text { It is crucial to define easily obtainable indicators that add value to } \\
\text { understanding the process. Do not waste time designing indicators that do } \\
\text { not add value to the process }\end{array}$ & \\
\hline $\begin{array}{l}\text { Step } \\
10\end{array}$ & Improvement routine & $\begin{array}{l}\text { Maintaining the improvement routine over time is the most difficult part of } \\
\text { the process as it requires full commitment from both the improvement } \\
\text { group and all staff involved in the process. It is important to design agile } \\
\text { mechanisms such as "Scrum" to monitor indicators. In the space of a short } \\
\text { time each day, this helps maintain motivation and raises awareness so } \\
\text { deviations from the ideal process can be detected }\end{array}$ & $\begin{array}{r}\text { Summary of ten-step } \\
\text { standardization } \\
\text { procedure for use in } \\
\text { lean improvement } \\
\text { implementation } \\
\text { processes }\end{array}$ \\
\hline
\end{tabular}

\section{Implementation methodology outcomes}

Over the three years, the process improvement project was being implemented, the performance indicators collected showed the evolution of changes in organizational culture and management.

The main outcomes of the implementation process were (1) the high degree of satisfaction felt by the health-care professionals involved and (2) the commitment from the director of the center toward promoting process management using lean health-care methodology, and constructing and implementing of the ten-step project. Changes in organizational structure toward process management are complex, and therefore need a lengthy time frame so that 
procedures can be learned and adapted. Staff in the surgical unit were able to understand the change and adapt to it successfully, improving highly relevant surgical indicators.

If we focus on activity indicators and resource efficiency alone, the surgical units at the hospital, together with the outpatient surgery unit, increased activity by an average of $8.4 \%$ per year for three consecutive years, which significantly reduced surgery waiting times. This output was achieved without increasing resources or hiring additional surgical staff.

Between July 2017 and July 2018, the number of outpatient operations in the surgical unit rose from 5,947 to 6,627, showing an increase of $11.4 \%$ in just one year. By contrast, the number of operations in the General Hospital during the same time period only rose from 4,221 to 4,399 . This means that only 78 more patients underwent major surgery using the same number of operating rooms as the previous year, showing an annual rise of $4.2 \%$ in a high-performance surgical block.

Both these increases are a direct result of the improvement process, which was carried out over three years using lean management techniques, and a direct result of the improvement projects, which were aimed at managing demand by adapting it efficiently to the surgical program. This included optimizing the use of time within the surgical unit, ensuring scheduled operations started punctually, reducing or eliminating patient cancelations and/or rescheduling, managing patients efficiently in the pre-admission areas, optimizing operating room turnover between surgical interventions and standardizing intra-surgical processes regarding material and equipment so that everything that is needed for the operation is ready and everything that does not add value to the process is removed.

Figure 2 shows the average delay in start times (in minutes) for all planned surgeries in the 19 operating theaters at the General Hospital for 2017, the first operation being scheduled at 8.30 a.m.

The chart shows that the trend toward obtaining efficient operating room use is close, not only in better time-keeping at the start of operations, but also in terms of preparing the operating rooms, rapid patient turnover and making the most of dead time between the operating theater's morning and afternoon shifts.

The surgical process indicators highlight increased activity as well as monitoring the performance and use of the operating theaters and all incidents that may affect the patient's safety.

The ten-step method was used to structure the process improvement team of surgical process of Vall d'Hebron Hospital and three other Spanish hospitals. The overall results of implementing the method have been very positive. They can be maintained over time, and they also help the hospital to better manage the surgical demand, as well as reducing patient waiting times.

Figure 2.

Minutes of delay at the start of surgical operations from 8.30 a.m. onward

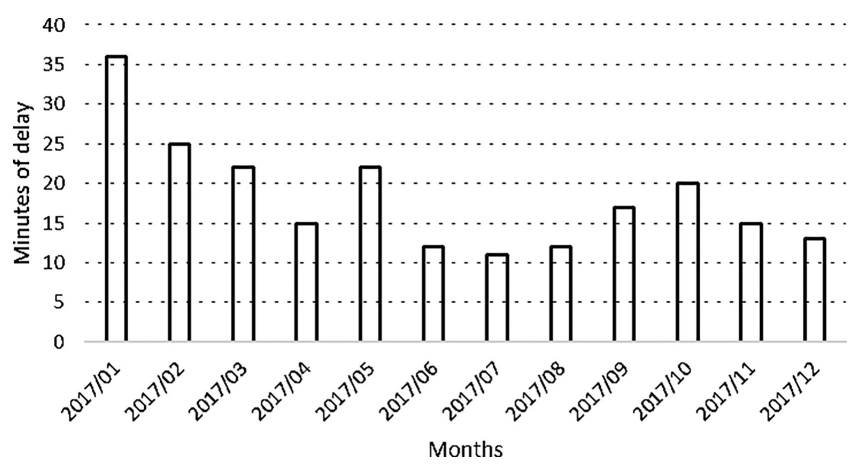




\section{Conclusions}

The method developed in this implementation helps bridge the gap in the literature pointed out by Bonome et al. (2016) regarding knowledge on improvement processes within the health sector based on lean principles. Using action research methods, it has been possible to intervene in the process and take the steps needed to draw up a roadmap for implementing lean management in health care in a way similar to traditional manufacturing environments, where the use of lean six sigma method is more usual (Black, 2007; Netland, 2016; Sahoo and Yadav, 2018; Shah et al., 2008; Improta et al., 2019).

Throughout the implementation, the critical factors and barriers found in the literature have been taken into account, and the resulting ten-step method was developed to initiate and consolidate a lean transformation process in a hospital environment.

The implementation has meant a large deployment of improvement projects in all the hospital operating rooms. These projects have contributed significantly to improving the activity and efficiency indicators of surgical resources and can be maintained over time. The teams of health-care professionals who initiated the first lean projects have maintained the continuous improvement routine. They have also been able to further develop the surgical process to achieve a lean transformation at all levels and all within an environment where the patient always comes first.

The ten-step methodology has been used in other surgical areas of the hospital to improve patient flow. The result has been a clear increase in activity because of the reduction in wasted time and better coordination between the health-care professionals. Lean methodology has not only led to improved performance, it has also brought the group together and consolidated adherence to procedures, ultimately leading to a cultural shift in the way new improvement projects at the hospital are approached.

Insights gained from this lean implementation improvement project can be applied to any hospital initiating a transformation project and requiring a new management approach. The ten-step process provides a clear and feasible methodology to implement change in way that involves with all health-care staff in the process and can be used to make real improvements in patient flow and communication between professionals, both of which ensure patient safety and improved resource efficiency.

\section{References}

Akmal, A., Greatbanks, R. and Foote, J. (2020), "Lean thinking in healthcare - findings from a systematic literature network and bibliometric analysis", Health Policy, Elsevier Ireland, Vol. 124 No. 6, pp. 615-627, doi: 10.1016/j.healthpol.2020.04.008.

Antosz, K. and Stadnicka, D. (2017), "Lean philosophy implementation in SMEs - study results", Procedia Engineering. doi: 10.1016/j.proeng.2017.03.107.

Baldassarre, F.F., Ricciardi, F. and Campo, R. (2018), "Waiting too long: bottlenecks and improvements - a case study of a surgery department”, TQM Journal, Vol. 30 No. 2, pp. 116-132, doi: 10.1108/ TQM-07-2017-0087.

Barnabè, F. and Giorgino, M.C. (2017), "Practicing lean strategy: Hoshin Kanri and X-matrix in a healthcare-centered simulation", TQM Journal, Emerald Group Publishing, Vol. 29 No. 4, pp. 590-609, doi: 10.1108/TQM-07-2016-0057.

Ben-Tovim, D.I., Bassham, J.E., Bolch, D., Martin, M.A., Dougherty, M. and Szwarcbord, M. (2007), "Lean thinking across a hospital: redesigning care at the Flinders Medical Centre", Australian Health Review: A Publication of the Australian Hospital Association, Vol. 31 No. 1, pp. 10-15, doi: 10.1071/AH070010.

Black, J. (2007), "Design rules for implementing the Toyota production system", International Journal of Production Research, Vol. 45 No. 16, pp. 3639-36641, doi: 10.1080/00207540701223469. implementation in a surgical

unit 
Bonome, L., Costa, M. and Filho, M.G. (2016), "Lean healthcare : review , classification and analysis of literature", Production Planning and Control, Vol. 7287, pp. 1-14, doi: 10.1080/09537287.2016. 1143131, Taylor and Francis.

Bortolotti, T., Boscari, S. and Danese, P. (2015), "Successful lean implementation: organizational culture and soft lean practices", International Journal of Production Economics, Elsevier, Vol. 160, pp. 182-201, doi: 10.1016/j.ijpe.2014.10.013.

Burgess, N. and Radnor, Z. (2013), "Evaluating lean in healthcare", International Journal of Health Care Quality Assurance, Vol. 26 No. 3, pp. 220-235, doi: 10.1108/09526861311311418.

Chung, R.D., Hunter-Smith, D.J., Spychal, R.T., Ramakrishnan, V.V. and Rozen, W.M. (2017), "A systematic review of intraoperative process mapping in surgery", Gland Surgery, Vol. 6 No. 6, pp. 715-725, doi: 10.21037/gs.2017.11.02.

Coughlan, P. and Coghlan, D. (2002), "Action research for operations management”, International Journal of Operations and Production Management, Vol. 22 No. 2, pp. 220-240.

D'Andreamatteo, A., Ianni, L., Lega, F. and Sargiacomo, M. (2015), "Lean in healthcare: a comprehensive review", Health Policy, Elsevier Ireland, Vol. 119 No. 9, pp. 1197-1209, doi: 10. 1016/j.healthpol.2015.02.002.

de Souza, L.B. (2009), "Trends and approaches in lean healthcare”, Leadership in Health Services, Vol. 22 No. 2, pp. 121-139, doi: 10.1108/17511870910953788.

de Souza, L.B. and Pidd, M. (2011), "Exploring the barriers to lean health care implementation", Public Money and Management, Vol. 31 No. 1, pp. 59-66, doi: 10.1080/09540962.2011.545548.

Gustavsson, S., Gremyr, I. and Kenne Sarenmalm, E. (2016), "Using an adapted approach to the Kano model to identify patient needs from various patient roles", TQM Journal, Vol. 28 No. 1, pp. 151-162, doi: 10.1108/TQM-04-2013-0050.

Henrique, D.B., Rentes, A.F., Filho, M.G. and Esposto, K.F. (2016), "A new value stream mapping approach for healthcare environments", Production Planning and Control, Taylor and Francis, Vol. 27 No. 1, pp. 24-48, doi: 10.1080/09537287.2015.1051159.

Hicks, C., McGovern, T., Prior, G. and Smith, I. (2015), “Applying lean principles to the design of healthcare facilities", International Journal of Production Economics, Vol. 170, pp. 677-686, doi: 10.1016/j.ijpe.2015.05.029.

Hu, Q., Mason, R., Williams, S.J. and Found, P. (2015), "Lean implementation within SMEs: a literature review", Journal of Manufacturing Technology Management. doi: 10.1108/JMTM-02-2014-0013.

Improta, G., Balato, G., Ricciardi, C., Russo, M.A., Santalucia, I., Triassi, M. and Cesarelli, M. (2019), "Lean Six Sigma in healthcare: fast track surgery for patients undergoing prosthetic hip replacement surgery”, TQM Journal, Vol. 31 No. 4, pp. 526-540, doi: 10.1108/TQM-10-2018-0142.

Ingelsson, P., Bäckström, I. and Snyder, K. (2020), “Adapting a Lean leadership-training program within a health care organization through cocreation", International Journal of Quality and Service Sciences, Vol. 12 No. 1, pp. 15-28, doi: 10.1108/IJQSS-09-2019-0107.

Joosten, T., Bongers, I. and Janssen, R. (2009), "Application of lean thinking to health care: issues and observations", International Journal for Quality in Health Care, Vol. 21 No. 5, pp. 341-347, doi: 10.1093/intqhc/mzp036.

Kahm, T. and Ingelsson, P. (2020), "Stuck in the middle first-line healthcare managers' responsibilities and needs of support when applying lean", International Journal of Quality and Service Sciences, Vol. 12 No. 2, pp. 173-186, doi: 10.1108/IJQSS-06-2019-0088.

Kollberg, B., Dahlgaard, J.J. and Brehmer, P.O. (2006), "Measuring lean initiatives in health care services: issues and findings", International Journal of Productivity and Performance Management, Vol. 56 No. 1, pp. 7-24, doi: 10.1108/17410400710717064.

Leite, H., Bateman, N. and Radnor, Z. (2020), "Beyond the ostensible: an exploration of barriers to lean implementation and sustainability in healthcare", Production Planning and Control, Taylor and Francis, Vol. 31 No. 1, pp. 1-18, doi: 10.1080/09537287.2019.1623426. 
Lima, R.M., Dinis-Carvalho, J., Souza, T.A., Vieira, E. and Gonçalves, B. (2020), "Implementation of Lean in health care environments: an update of systematic reviews", International Journal of Lean Six Sigma. doi: 10.1108/IJLSS-07-2019-0074.

Lindsay, C.F., Kumar, M. and Juleff, L. (2020), "Operationalising lean in healthcare: the impact of professionalism”, Production Planning and Control, Taylor and Francis, Vol. 31 No. 8, pp. 629-643, doi: 10.1080/09537287.2019.1668577.

Machado, V.C. and Leitner, U. (2010), "Lean tools and lean transformation process in health care", International Journal of Management Science and Engineering Management, Vol. 5 No. 5 , pp. 383-392, doi: 10.1080/17509653.2010.10671129.

Netland, T.H. (2016), "Critical success factors for implementing lean production: the effect of contingencies", International Journal of Production Research, Vol. 54 No. 8, doi: 10.1080/ 00207543.2015.1096976.

Nicolay, C.R., Purkayastha, S., Greenhalgh, A., Benn, J., Chaturvedi, S., Phillips, N. and Darzi, A. (2012), "Systematic review of the application of quality improvement methodologies from the manufacturing industry to surgical healthcare", British Journal of Surgery, Vol. 99 No. 3, pp. 324-335, doi: 10.1002/bjs.7803.

Niemeijer, G.C., Does, R.J.M.M., De Mast, J., Trip, A. and Van Den Heuvel, J. (2011), "Generic project definitions for improvement of health care delivery: a case-based approach", Quality Management in Health Care, Vol. 20 No. 2, pp. 152-164, doi: 10.1097/QMH.0b013e318213e75c.

Papadopoulos, T., Radnor, Z. and Merali, Y. (2011), "The role of actor associations in understanding the implementation of Lean thinking in healthcare", International Journal of Operations and Production Management, Vol. 31 No. 2, pp. 167-191, doi: 10.1108/01443571111104755.

Parkhi, S.S. (2019), "Lean management practices in healthcare sector: a literature review", Benchmarking, Vol. 26 No. 4, pp. 1275-1289, doi: 10.1108/BIJ-06-2018-0166.

Perona, M., Saccani, N., Bonetti, S. and Bacchetti, A. (2016), "Manufacturing lead time shortening and stabilisation by means of workload control: an action research and a new method", Production Planning and Control, Taylor and Francis, Vol. 27 Nos 7-8, pp. 660-670, doi: 10.1080/09537287. 2016.1166283.

Poksinska, B. (2010), "The current state of lean implementation in health care: literature review", Quality Management in Health Care, Vol. 19 No. 4, pp. 319-329, doi: 10.1097/QMH. 0b013e3181fa07bb.

Reed, J.E. and Card, A.J. (2016), "The problem with plan-do-study-act cycles”, BMJ Quality and Safety, Vol. 25, pp. 147-152, doi: 10.1136/bmjqs-2015-005076.

Rother, M. and Shook, J. (2003), Learning to See. Lean Enterprise Institute (LEI), Cambridge Center, Cambridge.

Sahoo, S. and Yadav, S. (2018), "Lean implementation in small- and medium-sized enterprises", Benchmarking: An International Journal, Vol. 25 No. 4, pp. 1121-1147, doi: 10.1108/BIJ-022017-0033.

Schattenkirk, D. (2012), "Building sustainable internal capacity for quality within a healthcare environment”, TQM Journal, Vol. 24 No. 4, pp. 374-382, doi: 10.1108/17542731211247391.

Shah, R., Chandrasekaran, A. and Linderman, K. (2008), "In pursuit of implementation patterns: the context of Lean and Six Sigma”, International Journal of Production Research, Vol. 46 No. 23, pp. 6679-6699, doi: 10.1080/00207540802230504.

Suárez-Barraza, M.F. and Miguel-Davila, J.A. (2020), "Kaizen-kata, a problem-solving approach to public service health care in Mexico. A multiple-case study", International Journal of Environmental Research and Public Health, Vol. 17 No. 9, doi: 10.3390/ijerph17093297.

Toussaint, J.S. and Berry, L.L. (2013), "The promise of lean in health care”, Mayo Clinic Proceedings, Vol. 88 No. 1, pp. 74-82, doi: 10.1016/j.mayocp.2012.07.025. 
Visintin, F., Cappanera, P., Banditori, C. and Danese, P. (2017), "Development and implementation of an operating room scheduling tool: an action research study", Production Planning and Control, Taylor and Francis, Vol. 28 No. 9, pp. 758-775, doi: 10.1080/09537287.2017.1310328.

Voss, C., Tsikriktsis, N. and Frohlich, M. (2002), "Voss et al. 2002”, International Journal of Operations and Production Management, Vol. 22 No. 2, pp. 195-219.

Womack, J.P. and Jones, D.T. (1996), Lean Thinking: Banish Waste and Create Wealth in Your Corporation, Simon \& Schuster, London.

Womack, J., Jones, D. and Roos, D. (1990), The Machine That Changed the World: The Story of Lean Production, Toyota's Secret Weapon in the Global Car Wars That Is Now Revolutionizing World Industry, Free Press, New York.

Yin, R. (2003), Case Study Research, Design and Methods, Sage Publications, Thousand Oaks, California, CA.

Zidel, T. (2006), A Lean Guide to Transforming HealthCare, Milwaukee (Wisconsin): American Society for Quality, Quality Press, Milwaukee.

About the authors

Marc Sales is an Industrial Engineer and a PhD Student in the Department of Business Organization, Management and Product Design, University of Girona, Girona, Spain. Marc has experience in research, development and implementation of operational excellence and continuous improvement systems on surgical processes in several hospitals in Spain. He was a Senior Consultant in companies in the health industry and Deputy Director of Processes and Innovation in Vall d'Hebron, Barcelona, Spain. His areas of expertise and interest are production planning control, lean health care and manufacturing and information technology (IT) solutions for the improvement of the surgical process.

Dr Rodolfo de Castro, PhD, is a Professor at the University of Girona. He is a Full Professor in the Department of Business Organization, Management and Product Design, University of Girona, Girona, Spain. His teaching is related to Operations Management at graduate and master's level. His research is focused on lean thinking in production and operations management and in supply chain management. He belongs to ADINGOR (Spanish Association of Engineers of Organization) and has participated in many competitive projects and collaborative projects in company. He has more than ten articles published in ISI journals. Rodolfo De Castro is the corresponding author and can be contacted at: rudi. castro@udg.edu

For instructions on how to order reprints of this article, please visit our website:

www.emeraldgrouppublishing.com/licensing/reprints.htm

Or contact us for further details: permissions@emeraldinsight.com 